\title{
A New Approach for Multicast Routing in Wireless Mesh Networks
}

\author{
Mustapha GUEZOURI, Ali KADDOURI \\ Signal Image Laboratory, Department of Electronics, Faculty of Electrical Engineering, University of Science and Technology, P.O. \\ Box 1505, El M’Naouer, Oran, Algeria \\ mguezouri@yahoo.fr
}

\begin{abstract}
This paper focuses on the multicast routing in wireless mesh networks under the 802.11 s standard. This standard defines the HWMP (hybrid mesh network protocol) as the protocol of the routing but only unicast and broadcast. Such the standard defines the multicast routing as a goal among several and that the HWMP don't handle multicast messages, this reason motivates us to think about a solution to make the HWMP able to handle multicast routing. We try to resolve the problem by using the bases of MAODV (Multicast Ad-hoc on-demand Distance Vector) protocol for the reason that the HWMP is inspired from AODV (Ad-hoc On-demand Distance Vector) and the MAODV is just an extension of the AODV. The result shows that the scalability of the routing using different number of nodes in the network is excellent. For the mobility it can be agreed since the routers in mesh topology is almost static. Whereas, the rate of message control generated is still very high. In all, the HWMP become able to handle up the multicast messages.
\end{abstract}

Index Terms - Wireless mesh networks, IEEE 802.11s, mesh routing protocol, HWMP, MAC Multicast routing

\section{INTRODUCTION}

Recently, many departments, campus and other public building start using the WMNs (Wireless Mesh Network) technology due to its various advantages such as low cost and easy employment. The researchers start looking for a new view of WMNs where the IEEE 802.11 s draft was created as a group task for standardization of WMNs in July 2004 [1]. This new technology defines different architectures that give new look for developing of algorithms and protocols over it. The large usage of WMN is to allow many nodes to access to internet over one or many portal nodes. Whereas, WMNs make out all applications which use multicast routing like multicast TV, Video conferencing, and multi-player gaming through internet connexion because of the no existing of a multicast protocol defined by the standard to route multicast packets.
The HWMP is the specified protocol in $802.11 \mathrm{~s} \mathrm{draft}$ It can route unicast and broadcast packets. In the other side, multicast packets are handled in the same way of a broadcast packet [1][2]. This concept touches to the performance of routing because of the high consummation of bandwidth without a goal in the case of multicast routing packets. Consequently, HWMP doesn't have an effective multicast algorithm.

A different multicast protocols in Ad Hoc network [MANET] (mobile ad-hoc network) have been proposed such as MAODV and OLSR (Optimized Link routing protocol) and other protocols. The most used protocol is MAODV which is an extension of AODV to integrate multicast routing in $\mathrm{AD}$ HOC networks [3], [4]. The MAODV is a tree Based protocol. The ODMRP (On-Demand Multicast Routing Protocol) is another Mesh-based protocol. The most research about multicast routing focuses on layer three (IP Layer) but there are also others whose work over layer two (MAC Layer). This motivates us to look after a solution which extends the HWMP to perform the multicast routing with a good performance.

Different works were done about this subject and each one with its methods, in this paper, we propose a MAC layer multicast scheme for WMNs based on the IEEE 802.11s standard. Our solution is based on the MAODV protocol for many reasons that we will see it in follow. This let the HWMP to be extended without much modification in HWMP's mechanism and messages control. It may be not fully compatible with the standard but it will have a few changes in the messages control like PREQ (path request) and PREP (path reply). Further, a new messages control will be added. This mechanism let build a tree for à multicast group and maintain the links periodically with a specific message control. The mechanism will be explained later in this paper.

Our work will be presented in the following sections: In section II, we describe the IEEE 802.11s draft where we will see the different architectures; also, we will speak a little bit about HWMP protocol and its base of conception. Then in section III, we will look after our proposition, where we will describe how the hybridization should be done and what is the reason to choose the MAODV as the point of starting in this research. 
In section IV, we show our result of simulation and we will discuss them clearly in section $\mathrm{V}$. we finish this paper with a global conclusion.

\section{BRIEF DESCRIPTION OF 802.11s STANDARD}

Before starting speaking about our solution, it very important to explain briefly what is the $802.11 \mathrm{~s}$ standard and what is new in this about Architectures, terminology of the nodes and hardware used, the functionalities and mechanism of the protocol and practically the HWMP algorithm. And when we understand all that, we will be able to speak about the problem.

After almost six years, the standardization of WMNs was announced in February 2010 [5]. this standard define different architecture of topologies and it contain the following kind of node; mesh portal which allow users to connect to internet, mesh station which support mesh service as selection of path (routing) and mesh access which provide both: mesh and point access functionalities [1]. This first type of architecture is called infrastructural/backbone WMNs Figure 1. It is composed of mesh routers forming a backbone for the clients to communicate over the network. The backbone can be build using various radio technologies. Mesh routers are self-configured, self-healing links among themselves. The mesh routers can be connected to internet thanks to the gateway functionalities. This approach provides also a backbone for conventional clients and enables the integration of WMNs with the existing wireless networks through gateway/bridge's functionalities in mesh routers. This type of topology is much used in WMNs. The second architecture is called client figure 2, it provide peer to peer network among client devices. In this type of architecture, the client node performs routing functionalities in the same way that it provides application services so we do not need mesh routers [6]. Packets destined to a node hops through multiple nodes to reach the destination. The clients nodes are usually use once radio technology to communicate. In this architecture client node become less performs to their application service such routing and self-configuration. The third and last type of architecture is called hybrid, this architecture combines between the infrastructure/ backbone and client WMNs as shown figure 3. Mesh clients can access to the networks through mesh routers as well as directly meshing with mesh clients. The infrastructure provides connectivity to other network such as internet. The hybrid architecture is most applicable case.

The standard defines the HWMP protocol as the default path selection algorithm [7]. This protocol use the metric called airtime metric to choose the better link over many candidate links which take into account both: transmission bit rate and error rate. HWMP protocol combines between two based protocols and this hybridization give it a great performance for routing, the first is on demand and the second is proactive. The On
Demand Path Selection mode is always available wither the root mesh station is configured or not. It is a variation of Ad Hoc On-Demand Distance Vector (MAODV) protocol called MR-AODV (multi route AODV).

On demand mode, the path discovery starts when a node has data to transmit to a destination and it doesn't know the route to this destination. In this case, it broadcast a path request (PREQ) management frame for that destination. Each station, receiving PREQ creates a route to the destination and forwards it. When a node receive a PREQ and If the station has a valid routing information about the destination address requested or it is the destination itself requested, it generates a path reply (PREP) management frame. Many nodes can respond to the demand but just one which is the best path (best metric) will be chosen. When a link is broken, the path error management frame will be generated [7].

Further then, in the proactive mode, a single mesh station is configured to be a path tree root and which broadcast PREQs periodically. Each station receiving PREQ updates its path information to the root. So every station knows route to the root and root knows a route to every mesh station. If direct route to destination is unknown, the data will be forwarded to the root which is responsible to forward it to the final destination.

Usually, the usage of WMNs with backbone network is to allow access to internet from one or many portal nodes. As explained above, there is only unicast path selection algorithm in the draft and exactly in HWMP protocol but it does not define any multicast packet transmission routing. Since the demand for the multicast service increases continuously, it is positively necessary and very important to handle the multicast packet perfectly. As a result, we propose a new approach to extend HWMP protocol to make it able to handle packets with multicast destination over WMNs based on the IEEE 802.11s standard.

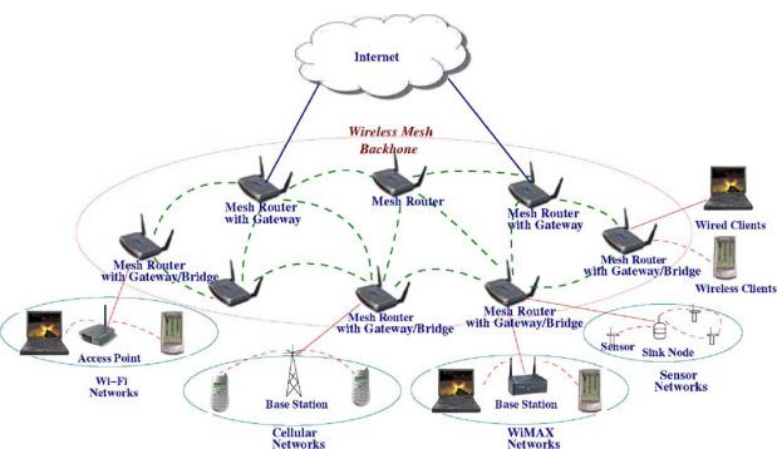

Figure 1: Infrastructure/backbone WMNs [11]

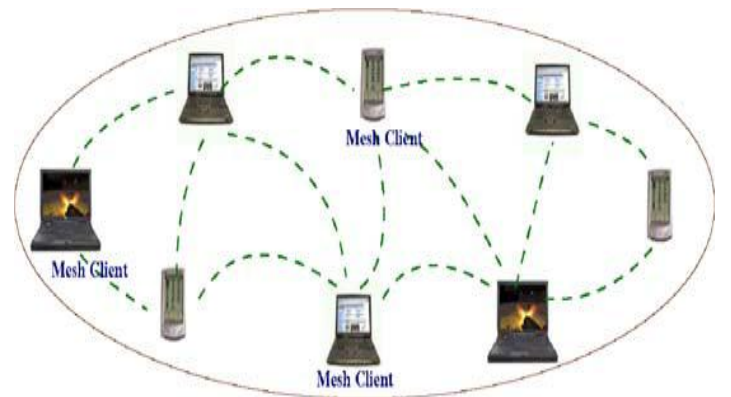

Figure 2: Client WMNs [11] 


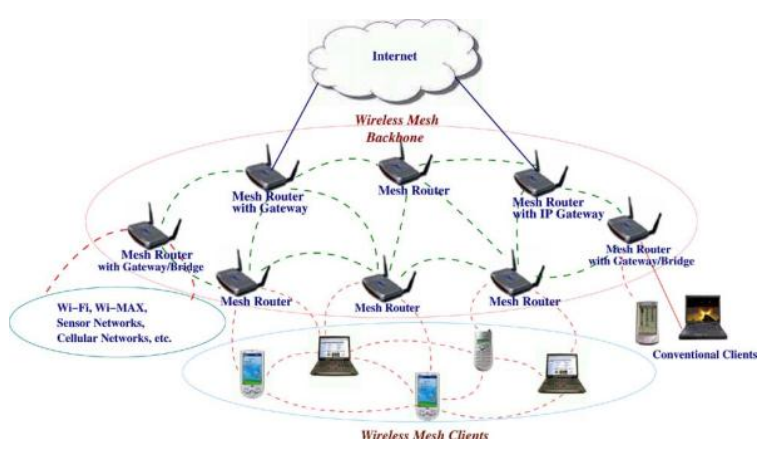

Figure 3: Hybrid WMNs [11]

\section{PROPOSED SOLUTION SCHEME}

Introduction:

After that we had seen the definition the concept and description of $802.11 \mathrm{~s}$ stand, it's time now to speak about our solution and reasons which make us to choose this idea as a proposed solution after that we had. Briefly, we will use the bases of MAODV protocol to resolve the problem of the multicast in HWMP and generally in the 802.11s standard

The goal of this paper is to propose a MAC layer multicast over HWMP protocol with minimal of modifications. Our proposed scheme is a kind of MAODV protocol that is adapted for layer 2 (MAC). The first goal to reach it is to allow multicast routing as well as the unicast routing, in meaning that we have to keep the HWMP principle as it is defined by the IEEE 802.11s draft. Even a few modifications will be required but this will change nothing in the operation mechanism. This is because the multicast and the unicast routing is speared by the condition marked through the destination MAC address and this is explained as follow; when a node has a frame to send. The first things to do is to read the destination address, in case that the destination is unicast or broadcast, it will be handled by the original HWMP mechanism without any change in it. Contrariwise, if the destination is multicast then it is handled with our scheme of multicast.

The question is: why did we choose MAODV and not OLSR, ODMRP or any other existing multicast protocol? .The answer is that HWMP is inspired from AODV protocol and MAODV is an extension of AODV which means that the use of MAODV look appropriate for the problem compared to other protocols. This deduction will show us some of similarities' features between the HWMP and MAODV. The first argument is that each one is a tree base routing. For HWMP, it generates a tree when a root node existed which is the same with MAODV that generate also a tree multicast group with a leader node manager. The second common feature is that both HWMP and MAODV use the same message frame control like path request (PREQ) and path reply (PREP). It is true that there is difference in the size of message frame control in each protocol because in HWMP, the PREQ contain a list of destination information unlike MAODV which contain only one destination by PREQ frame. It looks easy to look after this problem and then we can use only one instead of using many destinations as list. This is a particular case. And the third argument is that MAODV is the most used in Ad Hoc networks. The defect of MAODV using is in the structural/backbone architecture where routers have less mobility and that only client nodes are mobile. In this case, an undesirable overflow of message control for maintaining the multicast links. That can be decreased by a humoristic calculate which define the perfect time for rebroadcasting to maintain the multicast group tree.

There is another alternative to resolve this problem which is based on the exploitation of the HWMP tree for multicast routing. This possibility can be used respectively when a node is configured as root usually; this node is the point access which transfers from internet to local network nodes a multicast flux like the video streaming. The defect of this hypothesis is when the leader of the multicast group is not the root in the mesh network because the better link metric of the tree is from the nodes to the root node and not to the leader multicast group So that decrease the performance of time response and the good exploitation of bandwidth through the network. This idea is still able to be studied and gives it another look.

Lets now speak about the mechanism of this scheme to build multicast routing information (group multicast tree) and then maintain of the links (tree). There are two principal methods used, the first is to join a multicast group. So when a node wishes to join a group by its MAC group address, the node broadcast a PREQ message control and set the " $\mathrm{j}$ " flag in the PREQ. The destination address is always set to the multicast group address.

In case that the node knows the group leader and it has a route to it, the node may use the multicast group leader extension in the PREQ message control and then it place the group leader's address within and it unicast the PREQ to the corresponding next hop for that destination.

After transmitting, the node waits for the reception of a PREP. If the node does not receive PREP before the recommended delay and that the number of retries is not reached, it sends again a PREQ on broadcast.

If the node send on a unicast to a group leader and PREP is not received within a recommended delay. The node broadcasts subsequent PREQs for that multicast group across the network.

If no PREP is received after max retries, the node may assume that there is no other members of that particular group within the network.

If it wanted to join the multicast group, it then becomes the multicast group leader. Otherwise, if it only wanted to send packets without jointing the group, it drops the packets and aborts the session.

Before joining or sending a packet to a group, the node must first consult its group leader table. 
When a node receives a PREQ message, it can reply by PREP depending on " $\mathrm{j}$ " flag of the frame and it states. In meaning that it is a multicast tree member or multicast group member and also it depends on sequence member. The node check if the conditions are satisfied then it creates in the unicast, routing table the new entry or it updates the information routing and like that a reverse route is built which will be used to forward on unicast the PREP frame from the generator node to the source node requester. In the end of this phase, the source node which request for a multicast route will choose the best link with the best metric link. After that, the node sends a MACT (Multicast Activation) message to activate the link created by prep frame.

The second principal operation is when a node wishes to leave the multicast group. This mechanism is as follow;

In the beginning, the node checks if it is a group leader. If true, it checks after how many multicast neighbors exist in its multicast table. If there is only one multicast neighbor for this node then the node leave the group and send a MACT with $\mathrm{p}$ flag set to 1 to his neighbor. Else, if there are many multicast neighbors, it select then one of his these multicast neighbors and then it leaves the group but it stays like a multicast tree member. After that, it sends a MACT message with $G$ flag $=1$ then it changes the direction of the selected router, from Downstream to Upstream at the reception of the MACT message by another nodes, the node check up the MACT4s Flags, if $\mathrm{p}$ is set and that the node is a group member and that it received the MACT message from Upstream neighbor then it become the new leader of multicast group and start broadcasting the group hello (GRPH: Group Hello ) message periodically. The first group hello message will be broadcast with a flag $\mathrm{U}=1$ to inform that there are a change is the multicast tree information and that a new leader is selected.

If the node is a router or tree member, it checks up if it has many downstream neighbors. In this case, the node select one of their neighbor and then it send a MACT with flag $\mathrm{G}=1$, whereas, the node leaves simply the tree multicast.

In case that a node receives a MACT with G flags is set to 1 ,it changes the direction of its neighbor from upstream to downstream and after that, it check it is member. In true case, it becomes a group leader and then it process in the same way described above. In the contrary case, it forwards the MACT message.

After these two principal operations of jointing and leaving a multicast group, a third operation is maintaining of link or maintain of the multicast tree.

We proposed a mechanism to maintain a link and repairing it. This mechanism is as follow; When a node joints a group, it defines a time delay called RETRANSMIT_TIME. During this period, if the node doesn't receive any message from its neighbors. It checks up the variable or flag "second". If this variable isn't set then it send a PREPACK (PREP
Acknowledgement) to this neighbor to look if the node is alive or not. This procedure is repeated on loop. In the case that "second" is set, the node start the repairing of the link.

When a node detect a broken link, it proceed in the same way to jointing a multicast group but the PRES is generated with a flag unset.

\section{SIMULATION AND DISCUSSION OF PERFORMANCE}

Introduction:

To improve the effect and result of our idea to integrate the multicast routing in HWMP, we will use the NS-3 simulator to simulate the differences algorithms which help to make the multicast treating working perfectly. we present in this section the result and we discuss them sequentially.

We start now the description of the set of simulation parameters effects on the performance. Then, we present the result of the simulation and discuss it.

The four constraints of simulation are the mobility speed, the density of the network and data traffics. Then, we add other particulars scenarios which influence on the protocol behavior such as the number of nodes which want to join the group, the number of message etc.

The simulation results gave by the NS3 (Network Simulator 3) simulator are presented in form of diagrams, curves and graphic tree to make the interpretation easier. For the performance evaluation, the simulation was done with NS3 simulator and it was conducted on a $\left(1000 \mathrm{~m}^{*} 1000 \mathrm{~m}\right)$ with 32 mesh nodes positioned in a grid scheme. The period of simulation is 100 seconds.

We see in this paper a simulation for each constraint as follow:

\section{A. Mobility constraint}

In this simulation, we are interesting by the influence of the nodes speed mobility on the multicast performance. The mobility speed takes the values: 0.1 , $1.1,1.5,4$ and $5 \mathrm{~m} / \mathrm{s}$. The number of nodes in the network is 32 in a surface of $1000 \mathrm{~m}^{*} 1000 \mathrm{~m}$

a. Results

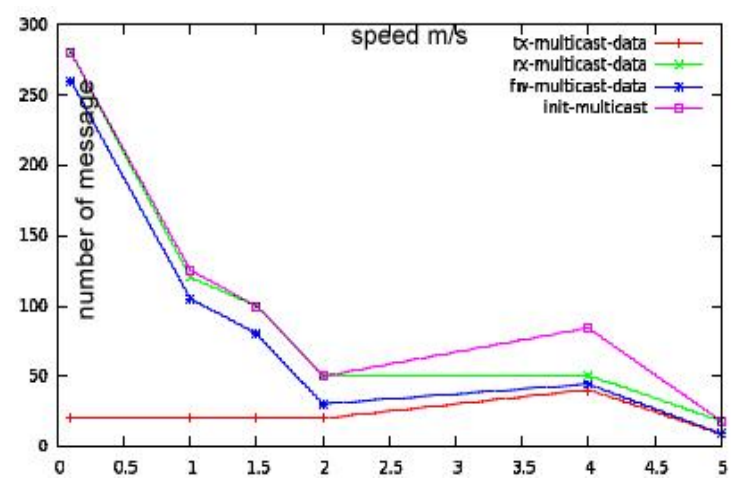

Figure 4: Curve of delivered message number on varying the mobility speed 


\section{b. Discussion}

In figure 4, the curves show that in the moment the mobility is almost null, the number of messages sent will be so high. That is the same with the forwarded messages and the reception of messages are all equal to $100 \%$. After, the curves start go down progressively each time when we increase the mobility speed except that the message sent are all received with a number less elevated then in a weak mobility speed. Also, the numbers of messages that must be send decrease. This event is explained by the no existing of an open session between the clients which move so quickly.

As $u$ can see, even the rate of the message sent decrease also. If the node can't send messages, it can be due to the no opening session between nodes and that event can be related to the latency of node to open and control an open link between two nodes so, we have to take a look to the algorithm latency related to the controlling and opening of a link between nodes.

The multicast protocol presents a good deliverance of multicast packets when the speed mobility is low. Each time we increase the mobility speed. The number of the nodes which cover the zone is also a constraint of deliverance message success in the high mobility case.

Such usually, the routers are static, we can accept the result as it is. The performance of this algorithm still medium if we speak about the mobility constraint.

\section{B. network density constraint}

We take experimentation by varying the number from 10 to 32 . We set the nodes in group to three and the node which send multicast messages in only one node. The time internal of transmission between tow frames is $0.1 \mathrm{~s}$.

\section{a. Results}

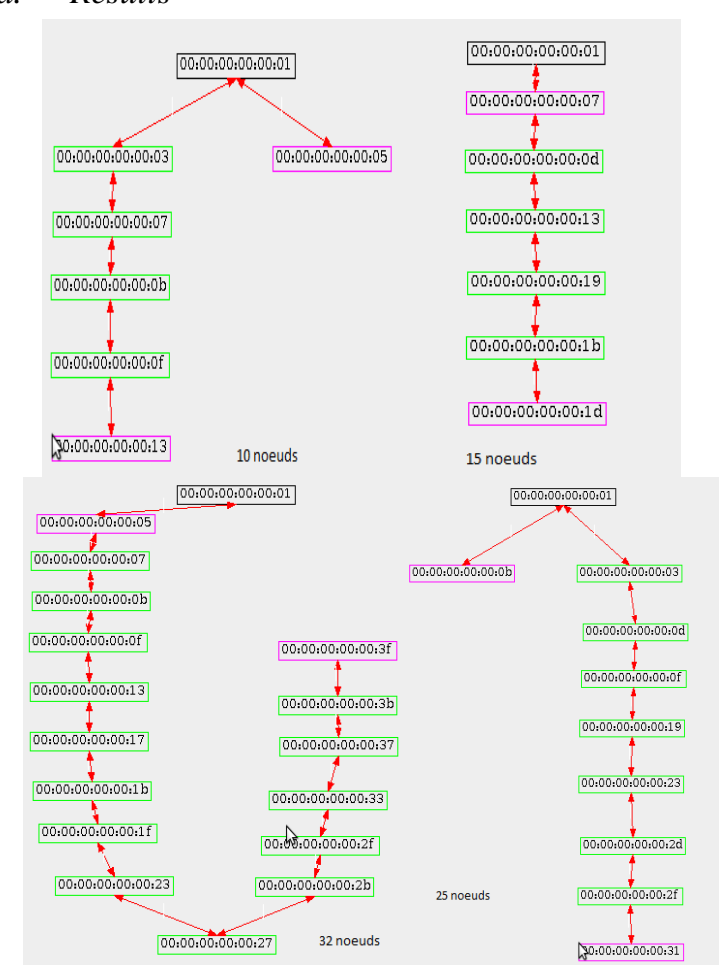

Figure 5: Routing trees by varying nodes number in the network

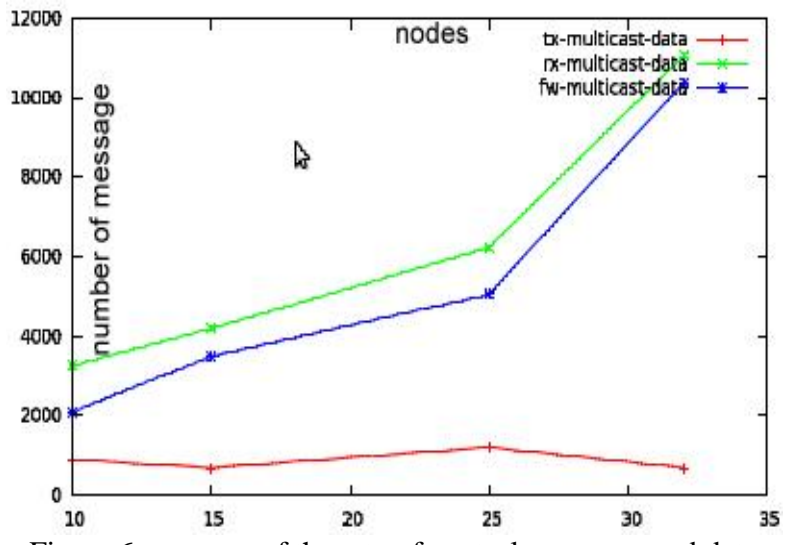

Figure 6.a: curves of the rate of control messages and data messages in the network.

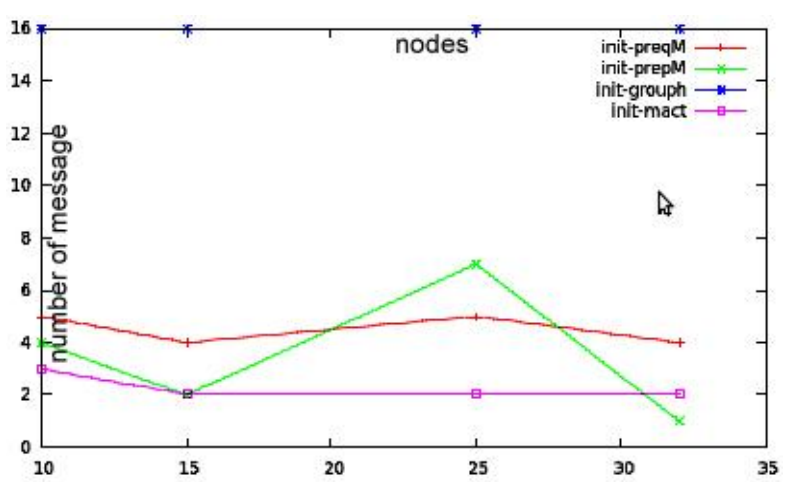

Figure 6.b: curves of the rate of control messages and data messages in the network.

\section{b. Discussion}

The figure 5 mentions that each time we change the number of nodes forming the network, the topology of the tree takes deferent forms. This is due to the different positioning of the nodes which join the group. From that, we deduce that the number of nodes in the networks influence on the tree topology. Further then, the number of the generated message depends directly of the number of nodes forming the networks and also those who form the multicast tree. As we see in the first curve in figure 6, the rate of message increase each time that we increase the number of the nodes exponentially in the case of 32 nodes. That is justified by the architecture of the routing tree which contains 16 intermediary nodes between the leader and the number of nodes situating in the end of the tree (leaf).

For the control messages, it's clear that the number of group hello messages is fixed (16 messages). That is explained by the unique selection of the group leader which generate periodically in the entire of network. While the number of PREP message initiated varies between 3 and 5 that depends on the number of nodes which join the group and the architecture of the multicast tree.

The multicast algorithm shows the success to build the multicast tree with deferent numbers of nodes in the network. This gives an appreciate result for the scalability over the number of nodes in the network. 
The number and the topology of nodes influence directly on the protocol behavior for the sending and the reception of multicast message and control message.

\section{C. simulation of operations}

a. Variations of nodes wishing join the group

In this scenario, we are interested by the number of nodes jointing the group effects on the multicast protocol. So, the variation is taken over the number of nodes which want to join the group, while the following parameters are static; Nodes which send the message during the simulation period and the time interval between twist message transmissions is 0.1 second.

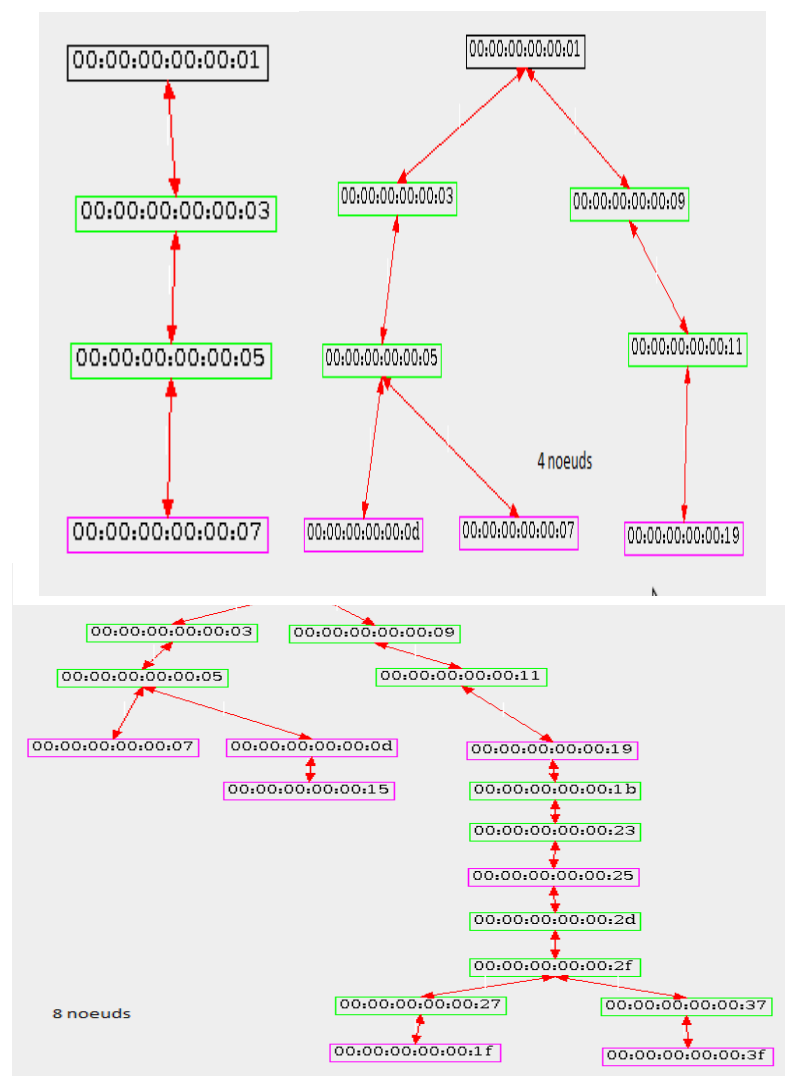

Figure 7: Routing tree for different nodes number jointing the group

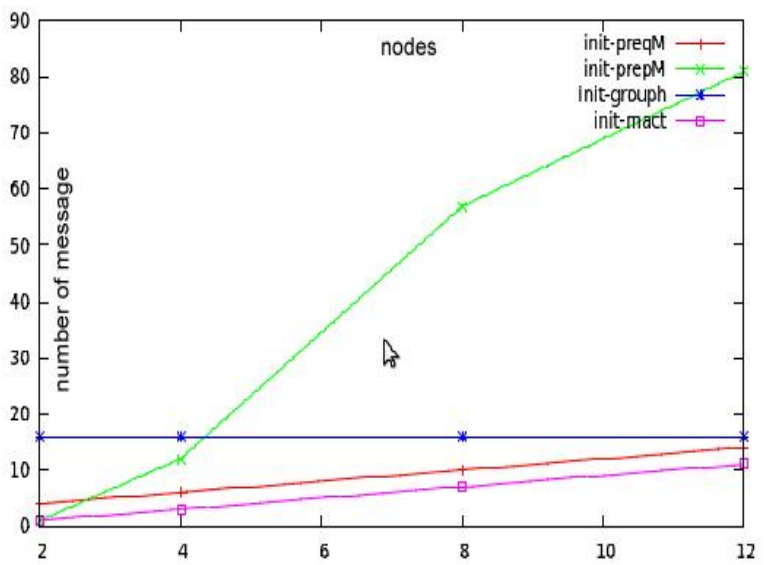

Figure 8: routing tree for different number of node jointing the group

\section{Discussion}

As for the rate of number of nodes in the network, it's clear that each time that the number of nodes jointing the group increase, the control messages increase in parallel. Further then, the multicast routing tree was more concerned by the variation of its architecture.

The multicast tree takes different architecture each time a new node joint the multicast group. The figure 8 presents the rate of different control message like PREQ, PREP, MACT and GRPH under a form of curves. We remark that the number of initiated PREP is more and more great, compared to the rest of message. The quick augmentation of PREP message is due to the multiple responses from one or many nodes of the network just like explained before. It's clear also that the number of group HELLO message is static in each simulation. That because after a selection leader, it begin to send GRPH periodically. Both PREQ and PREP message increase linearly, because each time that node want to join the group, it send a PRES for discovering the group. In instance, in the 12 nodes case, we see about twenty PREQs which were evident. The first node sends three PREQs, and it becomes a leader, where the other nodes find a route from the first sent request of PREQ. A particular case allows to avoid the sending of PREQ message to join the group that is happened when the node is a member of the tree.

We remark also that the MACT is almost equals to the number of PREP since each time that a node receive a PREP which is the generator of PREQ, it must send back the MACT message to the last node which answered to it.

The number of message control increase each time that the number of nodes jointing the group increases.

The group hello message is all fixed while the PREP increase quickly compared to the other message control [8], [9], [10].

\section{b. Variations of nodes that leave the group}

In this scenario, we study the impact of leaver nodes on the protocol and the control messages generated. For that, we put 10 nodes in the group, and then we increase the number of nodes which want to join the group from 1 to 5 .

\section{Results}

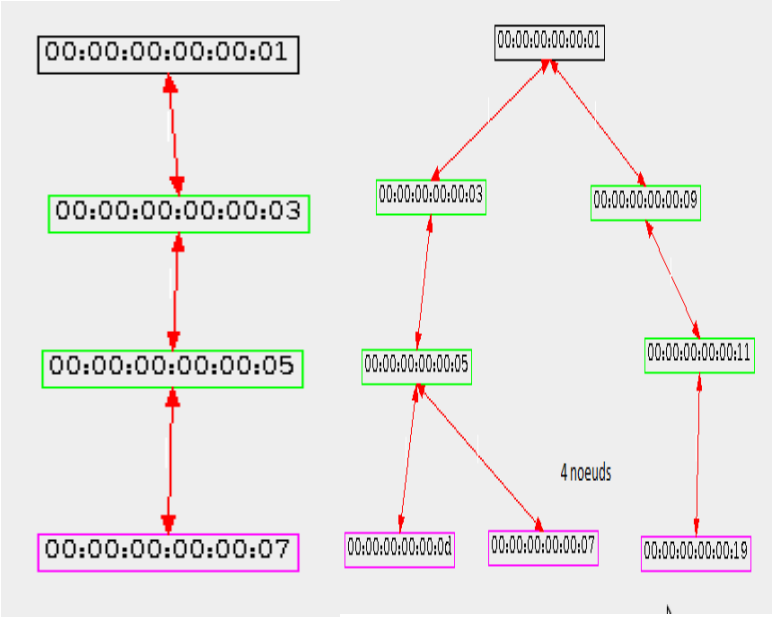




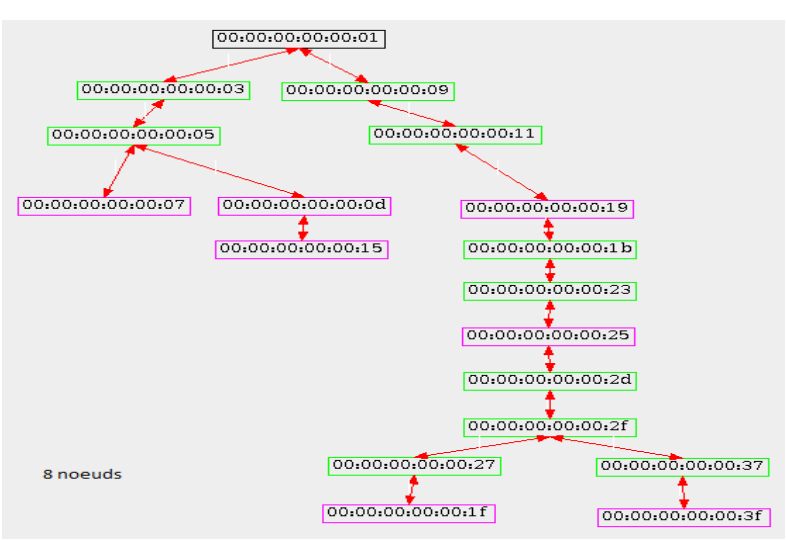

Figure 7. Routing tree for different nodes number jointing the group

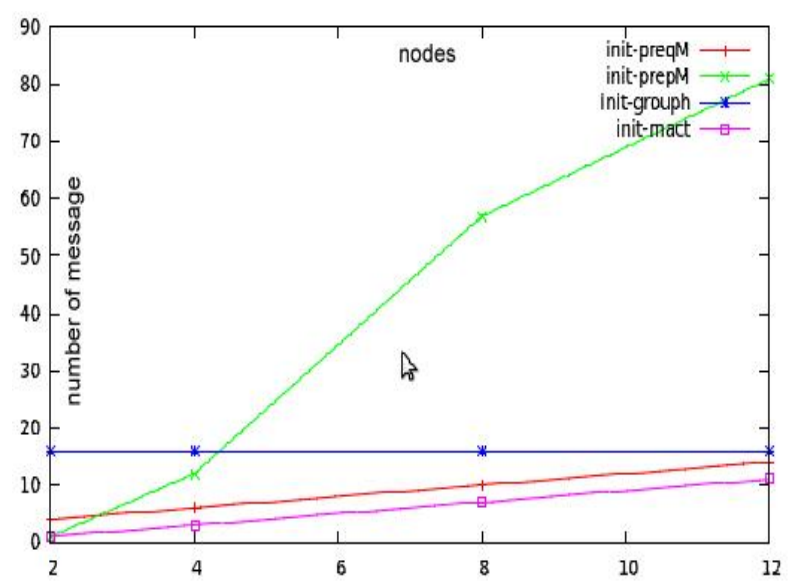

Figure 8: routing tree for different number of node jointing the group

\section{Discussion}

For the rate of number of nodes in the network, it's clear that each time that the number of nodes jointing the group increase, the control messages increase in parallel. Further then, the multicast routing tree was more concerned by the variation of its architecture.

The multicast tree takes different architecture each time a new node joint the multicast group. The figure 8 presents the rate of different control message like PREQ, PREP, MACT and GRPH under a form of curves. We remark that the number of initiated PREP is more and more great, compared to the rest of message. The quick augmentation of PREP message is due to the multiple responses from one or many nodes of the network just like explained before. It's clear also that the number of group HELLO message is static in each simulation. That because after a selection leader, it begin to send GRPH periodically. Both PREQ and PREP message increase linearly, because each time that node want to join the group, it send a PRES for discovering the group. In instance, in the 12 nodes case, we see about twenty PREQs which were evident. The first node sends three PREQs, and it becomes a leader, where the other nodes find a route from the first sent request of PREQ. A particular case allow to avoiding the sending of PREQ message to join the group that is happened when the node is a member of the tree.
We remark also that the MACT is almost equals to the number of PREP since each time that a node receive a PREP which is the generator of PREQ, it must send back the MACT message to the last node which answered to it.

The number of message control increase each time that the number of nodes jointing the group increases.

The group hello message is all fixed while the PREP increase quickly compared to the other message control [8], [9], [10].

c. Variations of nodes that leave the group

In this scenario, we study the impact of leaver nodes on the protocol and the control messages generated. For that, we put 10 nodes in the group, and then we increase the number of nodes which want to join the group from 1 to 5 .

\section{Results}

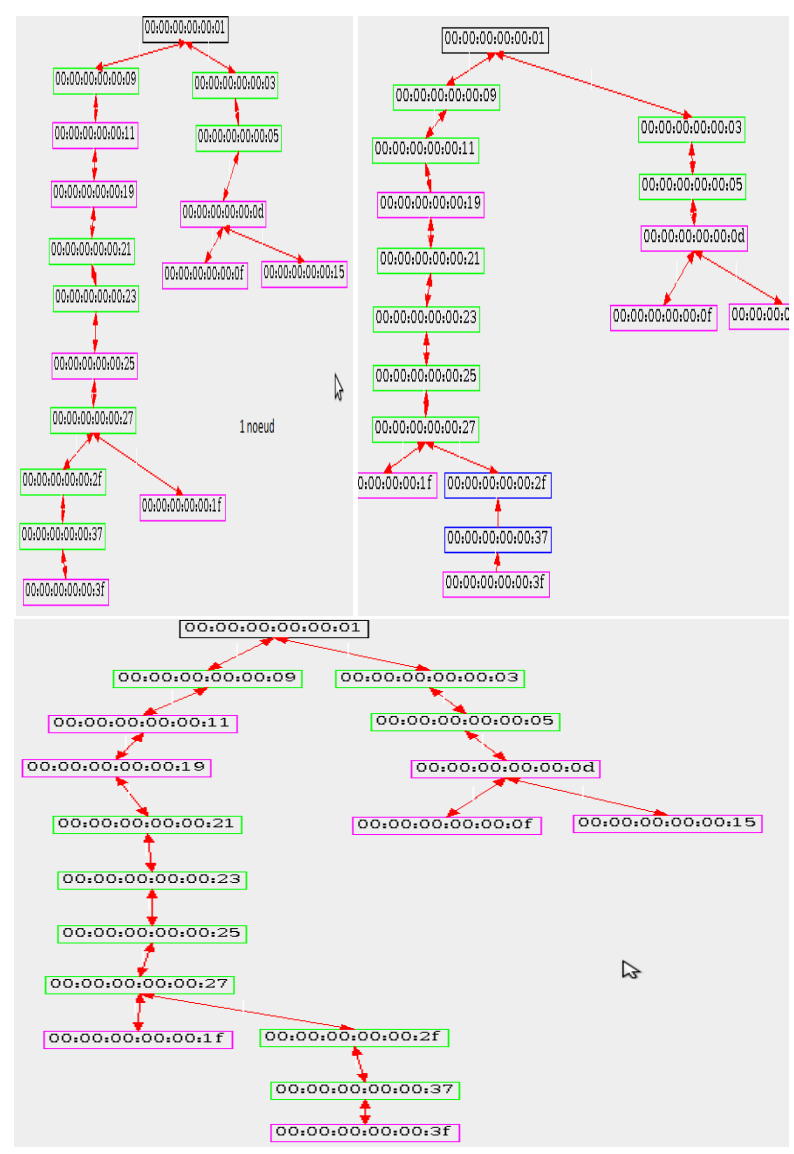

Figure 9: Different routing trees in each case of leaving node number 


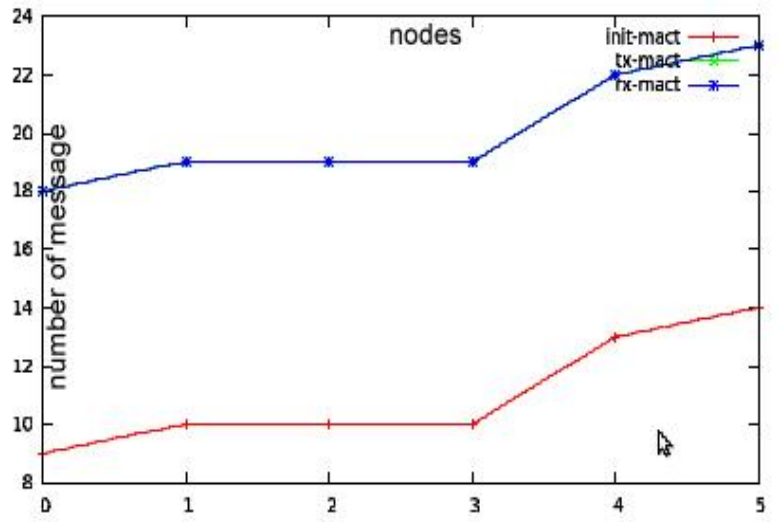

Figure 10: Curves of message control generation by varying the number of nodes leaving the group

\section{Discussion}

The curves illustrated in figure 10 shows the behavior of MACT message. We remark that the number of initiated PREQ go from 8, 13 to 14 . This is because of the using of MACT to leave from multicast group. For the twist curves tx-mact and init-mact we remark that it is on each other and it is evident since the number of message forwarder equals to the received by end and intermediate nodes of the multicast tree routing.

The figure 9 shows the multicast tree routing. It mentions the success of the leaving operation of a node from the group in the five cases and generally. It is nodes which were a group member and then it become tree member or it leave directly from the tree if it was a leaf of the multicast tree.

The leaving group constraint influence only on the MACT message generated.

\section{Particular case: the leader leaves the group}

It's possible that the multicast group leader want to leave the group. This particular case has to call to the whole specific algorithm. We present a particular scenario for this case, the simulation environment is the same with the one seen before. So, we take 8 mesh nodes, and then the leader tries to leave the group.

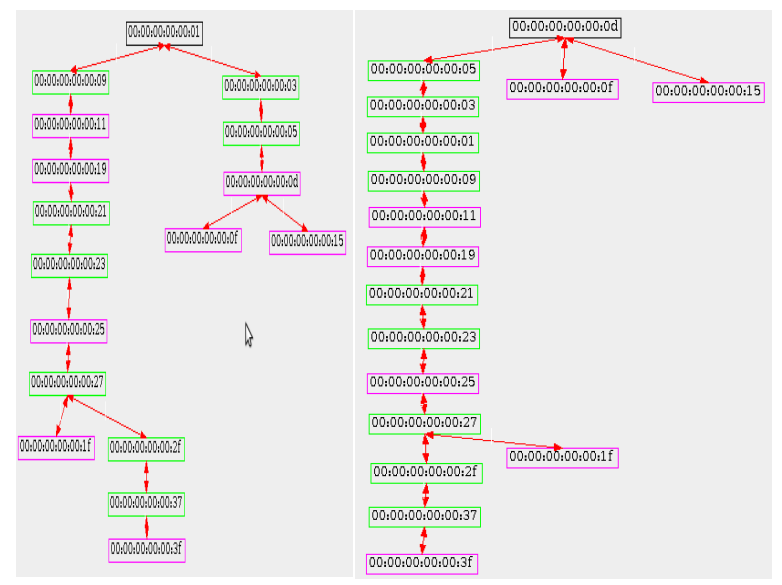

Figure11. Selection of new group leader

\section{Discussion}

The twist tree showed in the figure 11 present the selection of a new leader after getting order from the first leader. The particularity of this case is that the leader selects a new leader of the group before that it leaves this one. This step generates a MACT message control to select one of their neighbors. The node which was leader becomes a tree member. The new leader starts the sending of group hello message periodically and informing the network nodes of the changing happened in the tree information.

\section{V.CONCLUSION}

In this paper, we proposed a mesh multicast tree based algorithm to extend the HWMP protocol. Simulation result have shown a success and advanced of this scheme for deliverance of multicast message over a mesh networks. No more of new message added because of the similitude between the protocol MAODV and HWMP frames. As future work, we should add optional and give an adaptation of the MAODV protocol to operate dependence on the speed of mobility and the architecture used within and make it in real environment

\section{REFERENCES}

[1] Michael Bahr, "Update on the Hybrid Wireless Mesh Protocol of IEEE 802.11s". Siemens Corporate Technology, Information \& Communication, 2007

[2] Kirill Andreev, Pavel Boyko, "IEEE 802.11s Mesh Networking NS-3 Model", June 2011, Accessible via http:// 2010/dot11s.pdf

[3] Elizabeth M. Royer, "Multicast Ad hoc OnDemand Distance Vector (MAODV) Routing", Mobile Ad Hoc Networking Working Group INTERNET DRAFT, university of California, Santa Barbara, Accessible via http://tools.ietf.org/html/draft-ietfmanet-maodv-00 2000.

[4] T. Clausen, P. Jacquet, Network Working Group, Project Hipercom, INRIA, 2003

[5] Sung-Jun Bae and Young-Bae Ko, "Efficient Layer-2 Multicasting for IEEE 802.11s based Wireless Mesh Networks", September 2010, IEEE 978-1-42448086-9/10

[6] Ian F. Akyildiz a, Xudong Wang b, Weilin Wang, "Wireless mesh networks" ,Georgia Institute of Technologie, Atlanta USA, 2005

[7] Guido R. Hiertz, Sebastian Max, "Principles of IEEE 802.11s", metting network.

[8]: Sung-Jun Bae and Young-Bae Ko , "An Efficient Proactive Tree Building Scheme for IEEE 802.11s based Wireless Mesh Networks", Graduate School of Information and communication, Suwon, Republic of Korea 
[9]: Rui Campos, Carlos Oliveira, José Ruela, "WiFIX+: A Multicast Solution for 802.11-based Wireless Mesh Networks", INESC Porto and Faculdade de Engenharia, Universidade do Porto, 4200-465

[10]: Guokai Zeng, Bo Wang, Yong Ding, Li Xiao, Matt Mutka , " multicast algorithms for multi-channel wireless mesh networks", Department of computer science and enginnering, Michigan state university

[11]: Ian F. Akyildiz, Xudong Wang, Weilin Wang, "Wireless mesh networks: a survey", School of Electrical and Computer Engineering, Georgia Institute of Technology, USA, 1January 2005.

M.GUEZOURI was born in Algeria. He received the engineer degree from the national Algerian institute of Telecommunications (ITOran) in 1988. He received the Master and $\mathrm{PhD}$ degrees in electrical engineering in 1990 and 2007, respectively from the Science and Technology University in Oran. Since 1990, he has been a Research Assistant at Signal and processing Laboratory, University in Oran. He is currently an Assistant Professor of electrical and computer engineering at the University in Oran, Algeria. His current research interests include Signal processing, neural networks, mesh networks and computer security.

A.KADDOURI was born in Algeria. He received the Master degree from the University in Oran, Algeria in 2011. He is working toward the Ph.D. degree. His current research interests routing in mesh networks and cloud computing applications. 